\title{
Algunos resultados de la disponibilidad léxica de Resistencia: coloquialismos. Aplicaciones a la enseñanza de e/le
}

Hugo Roberto Wingeyer

Primera parte: el método del léxico disponible, ámbito de la investigación y el léxico disponible de Resistencia

\section{1- Introducción}

El desarrollo de programaciones relacionadas con la enseffanza del léxico a extranjeros requiere de la previa identificación de los vocablos de la comunidad de habla donde se va a planificar. El léxico disponible constituye un método estadístico eficaz para la identificación del léxico fundamental de diferentes comunidades.

En el marco de la metodología de la disponibilidad léxica, tomamos en consideración en este artículo aspectos una investigación que se está llevando a cabo en el Departamento de Letras de la Facultad de Humanidades de la Universidad Nacional del Nordeste, sobre la identificación del léxico de la ciudad de Resistencia, en el que se analizan los datos obtenidos de encuestas aplicadas a alumnos de escuelas secundarias en el año 2001.

En el campo de la lingúistica aplicada se han mostrado siempre interesados en encontrar un método estadístico eficaz para la identificación del léxico fundamental de diferentes comunidades de habla. Esta afirmación nos sirve de punto de partida tanto para fundamentar esta búsqueda de identificación del léxico, como para brindar información sobre el método utilizado en esta investigación, el método de la disponibilidad léxica.

Veamos ahora lo que nos aporta la siguiente cita: "Buena parte del conocimiento lingaístico de un hablante consiste en la información que ha internalizado sobre las palabras de su lengua. Cuanto más amplia sea, mayor posibilidad tendrá de establecer distinciones sutiles para captar la realidad en su variedad y dinamismo. Por otro lado, esta amplitud o conocimiento léxico depende de la experiencia individual, y del nivel sociocultural de los hablantes. A diferencia del conocimiento gramatical, que es compartido en su casi totalidad por los hablantes de una comunidad, hay mayores variaciones en el conocimiento linguístico que dependen de la experiencia individual y del nivel cultural de los hablantes." (Di Tullio, 1997:24)

Destacamos dos cuestiones: el papel decisivo del grado de conocimiento del léxico para percibir la realidad en toda su complejidad y los factores que hay que tener en cuenta cuando se intenta medir el conocimiento léxico de los hablantes.

\section{2- La disponibilidad léxica}

Volvemos al tema de la búsqueda de un método estadistico eficaz para la identificación del léxico, en nuestro se trata de la disponibilidad léxica. En este método se trabaja con el caudal léxico propio de una determinada situación comunicativa. Dicho más claramente, es el conjunto de vocablos usado en aquellas situaciones comunicativas especificas, determinadas por centros de interés o temas determinados: Partes del cuerpo, La ropa, Partes de la casa (sin los muebles), Los muebles de la casa, Alimentos y bebidas, Objetos 
colocados en la mesa para la comida, La cocina y sus utensilios, La escuela: muebles y materiales, Iluminación, calefacción y medios para airear o refrescar un ambiente, La ciudad, El campo, Medios de transporte, Trabajos del campo y del jardín, Los animales, Juegos y distracciones, Profesiones y oficios y Los colores. Muchos de estos centros constituyen campos semánticos de máxima rentabilidad en la enseñanza de $E / L E$.

Podemos afirmar que incluye tanto el léxico que usa, como el que conoce el hablante. Se basa en la aplicación de pruebas asociativas formadas por los mencionados temas o centros de interés en las que el informante vuelca todas las voces vinculadas con el mismo. Ahora bien, el indice de disponibilidad de cada uno de los términos surge de la combinación de la frecuencia y la posición que ocupa cada uno de ellos. Por último, en el método, los términos con los que trabaja se clasifican en palabras y vocablos, las primeras son todos los términos, repetidos o no; y los segundos, cada uno de los términos diferentes.

Para calcular la disponibilidad léxica se ha utilizado el programa LEXIDISP, disenado por José Enrique Moreno Fernández y Antonio García de las Heras, con el asesoramiento linguístico de Francisco Moreno y Pedro Benítez, y patrocinado por el Instituto Cervantes, La Universidad de Alcalá y la Asociación de Lingǘstica y Filología de la América Latina.

En cuanto a la aplicación de los resultados, por un lado a distintos aspectos de la Linguística (dialectológicos, sociolinguísticos, etnolinguísticos, semióticos) y, por otro, a la enseffanza-aprendizaje del espafiol como lengua materna y extranjera.

\section{3- El ámbito de la investigación. Resistencia}

En primer lugar, para la situación geográfica y caracterización linguística de la ciudad en estudio, aportamos información sobre la división del espafiol de Argentina que propone Donni de Mirande (1992:384-400), quien considera que el mismo forma parte de la comunidad hispanohablante y que no es uniforme sino que presenta diferencias, causadas por diversos factores: históricos, demográficos, culturales y socioeconómicos. Con respecto a las variaciones geograficas, ha establecido las siguientes zonas: 1. Litoral- pampeana (la casi totalidad de la provincia de Santa Fe, zonas de la de Entre Ríos, provincias de Buenos Aires, La Pampa y toda la Patagonia), 2. Nordeste (provincias de Misiones, Corrientes, este de las de Formosa y Chaco, nordeste de la de Santa Fe y norte de Entre Ríos), 3. Noroeste (oeste de las provincias de Chaco y Formosa, la mayor parte de Jujuy, Salta, La Rioja, Catamarca y Tucumán, excepto el centro), 4. Andina del norte (norte de la provincia de San Juan, oeste de las de La Rioja y Catamarca, extremo norte de Jujuy y parte del noroeste de Salta), 5. Cuyano-central (centro y noroeste de las provincias de Córdoba, centro y norte de San Luis, la mayor parte de San Juan, la provincia de Mendoza y norte de la de Neuquén), Santiago del Estero y regiones menores.

Nuestro trabajo se centra en el léxico de la ciudad de Resistencia, capital de la provincia del Chaco, situada, de acuerdo con las zonas presentadas, en la región nordeste, área de influencia de la lengua guaraní. Ahora, ¿cuáles son las características linguísticas generales de esta región del espafiol de Argentina? Se caracteriza, además de por la lógica presencia de rasgos comunes a otras áreas hispánicas, por la retención de modalidades tradicionales y por la presencia de la lengua aborigen, el guaraní, que genera interferencias morfosintácticas y fonético-fonológicas en el español.

Respecto del aspecto léxico, que es el particularmente nos interesa, el mismo se caracteriza, amén de la presencia de voces del español general, por el mantenimiento de voces 
tradicionales, descartadas en el litoral sur argentino y en otras áreas hispánicas, por préstamos guaraníes e identificaciones léxicas con voces guaranies.

Por último, brindamos información sobre aspectos socioeconómicos de esta ciudad que contaba, en el momento en que se realizaron las encuestas, en los meses de marzo y abril del año 2001, con una población de alrededor de 358.199 habitantes, de acuerdo con los datos estimados por la Dirección de Estadística y Censos de la Provincia del Chaco. Esta información nos permite afirmar que

Resistencia ocupa el $11^{\circ}$ lugar entre las ciudades argentinas [...], que constituye, junto con la ciudad de Corrientes, uno de los máximos centros urbanos del nordeste argentino, y que a través de las funciones que irradian organizan el espacio de las provincias del Chaco, Corrientes, Misiones, Formosa y norte de Santa Fe, conjuntamente con las ciudades de Posadas y Formosa. (Manoiloff, 2000:7)

\section{4- El léxico disponible de Resistencia \\ 4.1- Muestra}

Desde el punto de vista cuantitativo, los informantes son 501 alumnos de nivel secundario: 281 mujeres y 220 hombres. Las encuestas de aplicaron en 9 escuelas de la ciudad de Resistencia: 6 públicas y 3 privadas, 6 céntricas y 3 periféricas. Un primer conjunto de informantes, 242 alumnos del último curso: 138 mujeres y 104 hombres y un segundo conjunto, con 259 alumnos del primer curso: 143 mujeres y 116 hombres.

De acuerdo con los datos proporcionados por el Ministerio de Cultura y Educación de la Nación de la Argentina el número total de establecimientos de nivel medio o secundario en el Departamento San Fernando al que pertenece la ciudad capital de Resistencia era, en el año 2000 , de 51, 38 públicos y 13 privados; y el número total de alumnos de nivel medio o secundario 24.609 , de los cuales 22.416 correspondian a escuelas públicas y 2.193 a establecimientos privados. Esta es la información con la que contábamos en el momento en que disenábamos la investigación.

Si tomamos en consideración los resultados del Censo correspondiente al año 2001, vemos que el total de población de 13 años, que asistía a las escuelas, era de 5.200 , y de 17 afios, de 4.500; lo que da una población total en estudio de 9.700, es decir que nuestra muestra constituye el 5,16\% de ese total. (INDEC, 2001)

\section{2- Resultados}

Del procesamiento de las 501 encuestas surgió la información siguiente: un total de 123.436 palabras que se corresponden con 8.999 vocablos.

\section{3- Análisis de los resultados}

Sobre la base de los resultados del cálculo de la disponibilidad léxica, se analizan factores linguísticos y sociales. Respecto de los primeros, se identifican voces del español general de Argentina, neologismos, regionalismos correspondientes a la zona nordeste, objeto de nuestro estudio, coloquialismos, palabras provenientes de lenguas indígenas, extranjerismos, siglas, marcas comerciales y grupos léxicos. 
En cuanto a los factores sociales, se toman en cuenta las siguientes variables: sexó; escuela del centro y de la periferia, escuela pública y privada, nivel socioeconómico (alto, medio y bajo) y la edad de los informantes.

\section{Segunda parte}

\section{1- Introducción}

Como el objetivo de la comunicación es llamar la atención respecto de la utilidad de este tipo de investigaciones, tanto para el desarrollo de programaciones de enseffanzaaprendizaje del léxico, como para las actividades relacionadas con la ensefranza del mismo a extranjeros, consideraremos aquí un aspecto del análisis linguístico realizado: los coloquialismos. Presentamos una propuesta de acercamiento a estos vocablos en la que se combinan los niveles léxico y nocio-funcional.

Pretendemos alentar la introducción, de una manera sistemática, en la ensefianza de $\mathrm{E} / \mathrm{LE}$, de las muestras vivas ${ }^{1}$ que nos ofrecen este tipo de investigaciones, además de las muestras tomadas de textos literarios, periodísticos etc.

Hay, en España, investigadores que han considerado, para la enseñanza de espanol como lengua extranjera, los resultados de trabajos de disponibilidad léxica, obtenidos de hablantes nativos. Un investigador compara el léxico que aparece en diversos manuales de E/LE con los vocablos aportados por 257 estudiantes espańoles. Descubre la falta de criterio para la selección del vocabulario y considera el provecho que se puede sacar de estos estudios sincrónicos (Benítez, 1994). Otra estudiosa explicita la importancia del conocimiento del vocabulario disponible para una buena graduación de los contenidos léxicos a la hora de elaborar manuales de enseñanza de E/LE (Mateo García, 1994).

\section{2- Coloquialismos}

\section{1- Criterios tenidos en cuenta para su selección}

Se han identificado, sobre la base de la información que nos proporciona la bibliografia, y, ante la ausencia de la misma, nuestros conocimientos como hablantes de la modalidad linguistica examinada, la totalidad de expresiones coloquiales.

Antes de dar un concepto coloquialismo, tengamos presente esta observación de Mateo García (1996:52), respecto de los límites de la metodología del léxico disponible, comparada con metodologías que operan con léxico contextual, recogido en actuaciones reales. Refiere a "la neutralización de los factores pragmático-discursivos", por estar trabajada "a partir de listas elaboradas a partir de los datos elaborados por tests". Esta afirmación sobre la ausencia de estos factores linguísticos en la recogida de datos de la disponibilidad léxica, condicionaria nuestra observación de las expresiones coloquiales. Porque, partimos de la idea de coloquialismo, como una realidad de habla, una modalidad de uso, un registro'; continuamos con la clasificación en dos tipos de registros, el formal y el informal; y; terminamos con la identificación de coloquialismo con el registro informal. De todas maneras, es posible matizar los límites de la propuesta metodológica. Tengamos en cuenta, en términos de López Morales (1999: 11), que la disponibilidad léxica es "el caudal léxico utilizable en una situación comunicativa dada", recogido "a partir de unos estímulos verbales", que son los centros de 
interés. Por último, leemos en Briz (1998: 60), en su descripción del nivel léxico-semántico del espanfol coloquial, lo siguiente: "El léxico coloquial está constituido por un conjunto restringido del léxico común." Y, respecto de las unidades léxicas: "A pesar de que no puede hablarse de un léxico estrictamente coloquial, son relevantes ciertas frecuencias léxicas, preferencias o predominios léxicos, algunos de los cuales pueden estar marcados sociolectalmente." En nuestro trabajo, es más dificultosa la tarea de clasificar como coloquiales las unidades que no aparecen en la bibliografia consultada, o que sí aparecen, pero no clasificadas de esa forma. Lo que hacemos es analizarlas desde la perspectiva conceptual que acabamos de plantear, para poder así clasificarlas.

Se incluyen la palabra tabú y los fenómenos del eufemismo y del disfemismo'. De acuerdo con Moreno Fernández (1998:201-202), la primera refiere a "una palabra cuyo uso debe evitarse, generalmente por motivos sociales, politicos, sexuales, supersticiosos o religiosos: la palabra existe, pero su empleo provoca el rechazo o la recriminación de la comunidad o de un grupo social"; el eufemismo es "el recurso que la lengua pone a disposición del hablante para evitar el tabú"; y el disfemismo es la sustitución de "un término agradable, o simplemente adecuado, por otro ofensivo o peyorativo". En nuestra investigación: nos encontramos con la palabra tabuizada culo, su eufemismo cola; y como ejemplo de disfemismo, limón, con el significado de 'seno'. Continuando con términos tomados de nuestro trabajo, y como ejemplo de paralelismo semántico entre las palabras tabuizadas, las eufemisticas y las neutrales, presentamos, tal como lo hiciera López Morales', en su estudio sobre San Juan de Puerto Rico, las siguientes voces: picho, pito, pija, bolas, chilo, parte genital masculina, órgano masculino y pene. Estas voces presentan diferencias de uso que se fundamentan en divergencias sociales y pragmáticas.

Se incluyen en esta categoria las formas apocopadas porque presentan un matiz de menor formalidad'.

Se incluyen también los diminutivos'. De acuerdo con Alvar Ezquerra (1993: 57-58)', "en los sufjios diminutivos parece haber un sentido objetivo de aminoración, junto al que, o en su lugar, aparecen unos sentidos subjetivos del usuario, que introduce sus propios sentimientos en el discurso mediante esa posibilidad". Por ejemplo, en los términos saquito y blusita, recogidos (junto a saco y blusa) en el Centro 2, La ropa, creemos que existen connotaciones de significado que no tienen que ver con el sentido objetivo de tamaño. Más bien refieren, informalmente, a ropas utilizadas por personas muy jóvenes, entre otros sentidos posibles.

\section{2- Formas seleccionadas}

a) En el conjunto de los alumnos del último curso: carretilla, micromini, pupera, solerito, blusita, remerita, saquito, puperita, taquito, cascarudo, esperma de pitufo, séptimo regimiento, trago pantera, tetra, frito de papas, pantera rosa, disco, chorizo, concheto, negro, señora gorda, bolita, baranda, canillita, tortolitos, forro, truchada, minibús, a pata, fitito, en el dos, chata, boliche, teto, la piragua, sesenta y nueve, ñoqui, loca, busca, verde cotorra y negro piojoso.

b) En el conjunto de los alumnos del primer curso: pito, pija, bolas, goma, limón, chilo, paleta, pata, sobaco, hueso dulce, barriga, tanguita, gorrito. esperma de duende, champú, manuela, mochilita, gomita, trava, trola, tiburón, cuatri, chinita, guainita, reboleo, culear, coger indias, chupar pijas, micha, bambi, agachate que te pongo, chicas patoteras, culo 
sucio, hinchar las bolas, jodete, mi palo, orto sucio, chanta, manochanta, traficante de fasos, tachero, gato, cana, aputosado, negro de mierda.

c) En los dos conjuntos: cola, culo, concha, huevo, gamba, pera, cachete, picho, canilla, calzón, camperón, topsito, mini, radio, tele, fernando, birra, bici, chorro, boliche, trolo, loca, rancho, heri, prosti, zorro, guaina, bus, cole, bondi, coger, mirar tele, andar en bici, coger, tele, ring raje, compu, pasear en bici.

Una cuestión significativa en esta categoría es que en el segundo conjunto de informantes, el de los alumnos del primer curso, aumenta considerablemente la presencia de términos de registro coloquial. Ahora bien, sobre la base de la diferencia entre los registros coloquial y formal, podrían reconocerse dos modos de comunicación diferentes: el modo pragmático que "se adquiere de forma progresiva y natural por simple transmisión, se hereda por el simple contacto linguístico con otros hablantes" y el modo sintáctico que "se aprende a través de un proceso de enseñanza sistemático iniciado principalmente en la escuela". Si tomamos en consideración el grado de escolarización de cada uno de los conjuntos de informantes, el primero, a punto de terminar la escuela media y comenzar la universidad, y el segundo, comenzando la escuela media o secundaria, podemos ver que "de la evolución y desarrollo del modo sintáctico resulta el uso formal de la lengua; los del modo pragmático, el registro informal". Y, si consideramos ahora el comportamiento lingúístico de nuestros informantes:

Todo hablante que domina estos varios modos de expresión y es capaz de usarlos adecuadamente según el contexto decimos que es un hablante culto. Los hablantes que no han recibido, por ejemplo, una escolarización suficiente siguen utilizando el modo pragmático, el cual, incluso se refleja en su expresión escrita. (Briz, 1996: 24)

\section{3- Propuesta didáctica}

Nuestra propuesta tiene, para las expresiones coloquiales, dos instancias. Una, la de nivel léxico, en la que, con el auxilio de los diccionarios y estudios léxicos de que disponemos, tratamos de definir todas las unidades, sefnalamos siempre las diferencias que pudieran surgir del contraste entre nuestras observaciones como hablantes y conocedores del área linguística en cuestión y la información que nos brinda la bibliografia. Otra, la del nivel nocio-funcional, en la que, sobre la base de los términos que acabamos de definir y de algunas formas derivadas de los mismos, nos lanzamos a la búsqueda de algunos exponentes funcionales. Sólo pretendemos llamar la atención, con unos pocos ejemplos, sobre las dificultades que presenta la definición de las expresiones coloquiales cuando se combinan estas dos instancias.

3.1- Tomamos, en primer lugar, algunos coloquialismos de los centros 1,15 y 16, Partes del cuerpo, Juegos y distracciones y Profesiones y oficios.

A. Trabajo de nivel léxico: limón, chilo.

Centro 1, Partes del cuerpo: cola, culo, concha, picho, huevo, pito, pija, bolas, goma,

concha. Registrado en el DRAE con el significado de 'vagina'. 
picho. Recogido en los diccionarios de argentinismos consultados con el significado de 'pene'. El DRAE y el DUE no lo registran.

huevo. Es probable que este vocablo, registrado en el DRAE, fuera utilizado, en algún caso, por desconocimiento del término estándar: testículo.

cola / culo. Al vocablo culo, se lo considera tabú, por eso aparece en la posición menor y con un índice de disponibilidad más bajo que cola, que es el que con más frecuencia se utiliza con ese significado. Este fenómeno no escapa a la observación de los estudiosos del lenguaje en Argentina: "...culo, muy común entre las madres espanfolas, se escuda en Argentina bajo el apodo de cola." (Teruggi, 1978: 330)

EI DRAE recoge este uso de cola como argentinismo, precisando que se trata de un eufemismo de registro coloquial.

pito. EI DRAE lo recoge con el significado de 'pene'. Observamos que con idéntico significado aparece en Abad de Santillán y en Conde.

pija. El DRAE lo recoge, con el significado de 'pene'.

bolas. El DRAE no recoge este argentinismo que aparece en el DIHA con el significado de 'testículos'. Observamos que, en el espaniol de Argentina puede referirse tanto a los testículos como al organo genital completo.

goma. Este argentinismo se utiliza, según el DIHA y el NDArg, con el significado de 'seno', especialmente si éste es de gran tamaño. Observamos que en el área en estudio, tal como aparece en Conde, refiere a esa misma parte del cuerpo, con independencia del tamanio.

limón. No aparece en el DRAE este argentinismo que, de acuerdo con Conde, se utiliza con el significado de 'seno'.

chilo. Según el NDArg, este término refiere al 'pene'.

Centro 15, Juegos y distracciones: agachate que te pongo, chicas patoteras, culo sucio, hinchar las bolas, jodete, mi palo, orto sucio.

sexual'.

agachate que te pongo. Expresión utilizada con el significado de 'realizar el acto

chicas patoteras. Este grupo es un juego de palabras realizado sobre la base del nombre de la canción infantil "Chicas petiteras" y el término "patoteras".

culo sucio / orto sucio. Ambos grupos refieren a un 'juego de naipes en el que se van formando parejas de cartas, y en el que pierde el participante que se quedan con la carta que no tiene pareja', de acuerdo con la información que nos proporciona el DEArg. Esta misma fuente recoge el vocablo "orto", con el significado de 'ano'.

hinchar las bolas. Esta expresión se utiliza con el significado de 'molestar, fastidiar, importunar'. Teruggi recoge "hinchar" e "hinchabolas", con idéntico significado.

jodete. Respecto de este término, observamos que Conde recoge el vocablo "joder" con el significado de 'perjudicar, molestar', que es el que corresponde aquí.

Centro 16, Profesiones y oficios: chorro', ñoqui, loca, busca, chanta, manochanta, traficante de fasos, tachero, gato, cana.

chorro. Argentinismo de uso muy frecuente, que el DRAE no recoge. El NDArg propone dos definiciones que se ajustan al significado de esta palabra es el espaniol de Argentina: por un lado, "persona que se apropia ilícitamente de lo ajeno"; y por otro, "persona 
que cobra más de lo justo por una mercancía o servicio"; mientras que el DIHA, la presenta, sin más consideraciones, como sinónimo de ladrón.

ñoqui. Este argentinismo refiere, de acuerdo con la información que nos proporcionan el DRAE y el DIHA, a un 'empleado público que asiste a su lugar de trabajo solo una vez al mes, en fecha de cobro, es decir que, sin trabajar, cobra un sueldo'; en ambos diccionarios aparece con marcas de coloquialismo utilizado con intención despectiva.

busca. Este término, que no recoge el DRAE, aparece en el NDArg, con marcas de coloquialismo utilizado con intención despectiva, con el significado de 'persona que procura ganar dinero mediante trabajos fáciles u obtener beneficios de cualquier coyuntura que se presenta'.

chanta. De acuerdo con Conde, este vocablo se utiliza con el significado de 'persona que no paga sus deudas', el de 'insolvente moral', el de 'persona informal', y el de 'persona que gusta aparentar conocimientos, relaciones o ideas que no tiene'. EI DRAE no lo recoge.

manochanta. De acuerdo con Conde, es un grupo compuesto por "manosanta" y "chanta". El DRAE no lo recoge.

gato. 'Prostituta, en especial la refinada y cara.' Es el significado que propone Teruggi para este yocablo. EI DRAE no recoge esta acepción.

cana. 'Agente de policía', es el significado de este vocablo con el que nos encontramos en el DEArg. Aparece en el DRAE.

\section{B. Identificación de exponentes funcionales:}

Hablar de uno mismo y de los demás: Descripción física: cola, culo, goma. , Descripción de carácter: conchudo, huevón, caracúlico, boludo, patotero, cara de orto, hinchabolas, jodido, chorro, busca, chanta, chantapufi, cana. Descripción de aficiones y gustos: Saco fotos sólo para hinchar las bolas, no para dedicarme a eso profesionalmente.

Relacionarse con los demás: Valorar una opinión: ¡Qué huevada! Reaccionar negativamente: No hinchés las bolas.

Expresar sentimientos: Desesperación e impotencia: ¡Qué boludo que soy! Animar: Dale, huevón, no te desanimes ahora seguí adelante con todo. Alegría: ¡Qué suerte! ¡Qué culo que tenés! Sorpresa por no creer: ¡Qué chanta que es!

Presentamos estos exponentes, a manera de ejemplo, con la finalidad de reconsiderar los significados de los términos a la luz de los mismos. Es otro punto de vista para abordar el tema de los coloquialismos. Como es siempre necesario advertir sobre la adecuación del registro, es conveniente plantearse los niveles en los que van a ser considerados.

\section{Consideraciones finales}

Esperamos que esta comunicación sea sólo el principio de las muchas aplicaciones posibles del análisis de los resultados de la disponibilidad léxica de Resistencia, a la enseffanzaaprendizaje del E/LE. Y que, tanto estos resultados, como los de que en el futuro proyectamos realizar en otras comunidades linguísticas argentinas, sean una base coherente a la hora de programar la ensenanza del léxico a extranjeros. Nos gustaría además que estas investigaciones signifiquen, a mediano y largo plazo, una contribución importante al desarrollo de la ensefianza del E/LE en la Argentina. 